\title{
Distributed Control with Virtual Capacitance for the Voltage Restorations, State of Charge Balancing and Load Allocations of Heterogeneous Energy Storages in a DC Datacenter Microgrid
}

\author{
Runfan Zhang, Branislav Hredzak, Senior Member, IEEE, and Thomas Morstyn, Member, IEEE
}

\begin{abstract}
This paper proposes a distributed coordination control strategy for load sharing and energy balancing between heterogeneous energy storages. These control objectives are satisfied through a two-level control structure. At the primary level, the decentralized virtual impedance control, without the requirement of communication links, allocates the low frequency component of the loads to batteries, while the high frequency component is allocated to ultracapacitors. Distributed control strategy, introduced at the secondary level over a sparse communication network, achieves battery state of charge balancing and regulation of the local bus voltages. Two sets of data are exchanged via the communication links, the local bus voltages and state of charges of batteries. The distributed controller for the restoration of local bus voltages implements an average consensus protocol, while the controller for energy balancing uses a cooperative protocol. In addition, the ultracapacitor voltages are locally restored at a slower time-scale. The proposed control strategy is resilient to communication link failures and features plug-andplay capability. Presented results demonstrate performance of the proposed control strategy for an islanded 380 VDC datacenter with variable loads. Different operating conditions are verified through the RTDS Technologies real-time digital power system simulator using switching converter models and nonlinear battery models.
\end{abstract}

Index Terms-Heterogeneous energy storage systems, average consensus protocol, cooperative control, virtual impedance control, virtual capacitive control, state of charge balancing.

\section{INTRODUCTION}

$\mathbf{E}$ NVIRONMENTAL issues and the scarcity of fossil fuels motivate adoption of clean and renewable energy sources, such as wind, hydro and photovoltaic (PV) sources [1]-[3]. However, most of the energy sources are intermittent, depending on climate conditions, resulting in imbalance between sources and loads and hence affecting the power quality and reliability. Energy storage (ES) devices can improve the power quality and balance the mismatch between supply and demand, and provide a range of services such as: peak shaving, spinning reserve and strategic loading [4], [5].

Since many energy generation sources, such as PV, are DC, their integration with ES devices facilitates development of a DC microgrid. Compared with AC microgrids, DC microgrids

Runfan Zhang and Branislav Hredzak are with the School of Electrical Engineering and Telecommunications, University of New South Wales, Sydney, NSW 2052 Australia (email: runfan.zhang@student.unsw.edu.au; b.hredzak@unsw.edu.au;).

Thomas Morstyn is with the Department of Engineering Science, University of Oxford, Oxford OX1 2JD, U.K. (email: thomas.morstyn@eng.ox.ac.uk) require fewer power electronic devices, eliminate frequency and reactive power related issues, skin effects, provide higher voltage level, reduce energy losses and simplify control system design [6]. Among the most significant energy consuming loads in microgrids are LED lighting, variable-speed drives and datacenters [7]. Interconnecting these loads with the renewable energy sources and ES devices within the DC microgrid can improve the power efficiency and quality [8], [9]. Therefore, the DC microgrids are being widely implemented in remote areas, banks, hospitals and datacenters [10].

Different types of ES devices are suitable for different objectives [11]. ES devices with high power density, and slower response time, such as batteries and fuel cells, are suitable to provide steady load [12]. On the other hand, the ultracapacitors or supercapacitors can improve the peak power capacity of the microgrid, because of their high cycle life and fast response time, allowing them to compensate the peak power [13], [14]. This limits frequent charging/discharging of batteries, enhancing their lifetime through lower temperature rise, lower cycle rate and reduced depth of discharge [11], [15], [16]. At the same time, the lower energy capacity ultracapacitors do not respond to the large steady loads and hence are unlikely to run out of energy. Therefore, use of the heterogeneous ES devices in the microgrid is more compelling, than only homogeneous ES devices, as the advantages brought by complementary characteristics in energy and power density can be effectively exploited [16]. Hybridization of ES devices is an effective and economic solution providing fast dynamic performance, high power and large energy supply at the same time [17].

\section{A. Hierarchical Control Methods}

However, different dynamic characteristics of heterogeneous ES devices cause difficulties in control system design [18], [19]. Control of ES systems can be broadly divided, according to traditional hierarchical control system, into two categories: the primary droop control and high-level cooperative control. The droop control method, which is a decentralized control strategy, introduces a virtual resistance/impedance to the voltage control signal for the DC-DC converter to share loads proportionally. Although this control method does not cause any energy loss, it poorly shares the loads and regulates the voltage, since the resistance/impedance results in decrease of 


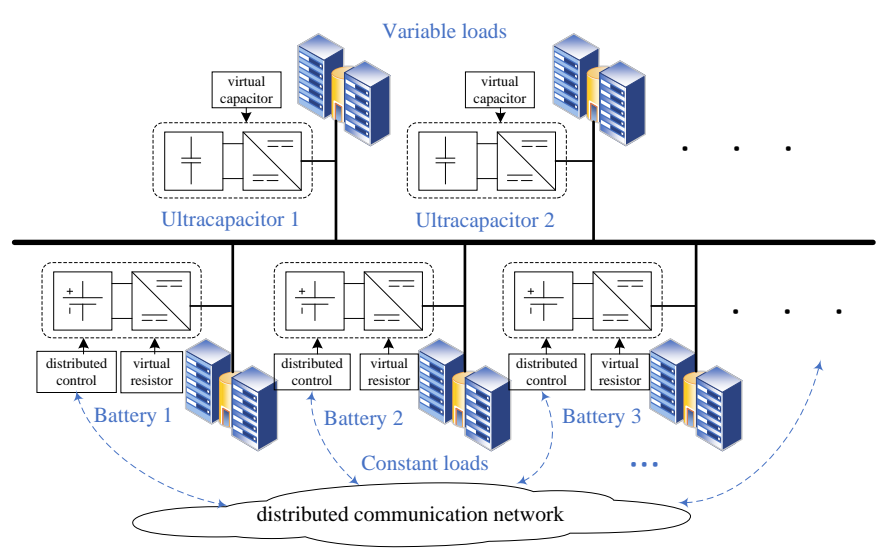

Fig. 1. Proposed control architecture for datacenter DC microgrid with distributed batteries and ultracapacitors to coordinate power sharing.

the local bus voltage [5]. Besides, because this control strategy fails to take the energy level of ES devices into consideration for sharing power, those ES devices with low energy level will prematurely run out of energy, which further deteriorates the power sharing performance.

\section{B. Droop Control Based Methods}

Modified droop control methods can mitigate the poor power sharing performance, but they cannot solve the problem completely [20]. Conventionally, two types of V-I droop control are applied to share power between each ES device: virtual resistance control [21], and impedance control [22]. The virtual resistance droop control, without the capability to allocate different frequency components of loads, can only be implemented in homogeneous ES systems alone. On the other hand, the virtual impedance control allows to allocate different frequency components of loads to specific ES devices [16], [17]. Most of the virtual impedance control methods are filter-based: a virtual resistance multiplying a high/low-pass filter to allocate loads [16], [23]. In contrast, [17] introduced a virtual capacitor, alongside with a virtual resistor, to assign the different components of the loads to heterogeneous ES devices. However, the local bus voltage deviation and poor load sharing issues remain. Consequently, the primary control method on its own is inadequate to control the distributed heterogeneous ES devices.

\section{Comparison of Centralized and Distributed Secondary Control Methods}

However, these problems can be solved at the secondary/tertiary level. The control systems at the higher level restore the microgrid voltage and provide load sharing based on overall distributed load in the microgrid. Conventionally, two types of control system are implemented in the microgrid: centralized or distributed cooperative control. The centralized control requires a full communication network [24]. On the contrary, a distributed control strategy tunes the control signal only based on neighboring ES systems' states and its local states [25]. In this case, the distributed control strategy only requires a sparse communication network, which provides robustness, extensibility and flexibility over the centralized method [26]. Both centralized and distributed control methods have already been introduced to ES systems [27]. Although these studies fix the voltage deviation problem and provide reasonable power sharing between ES devices, they have only been applied to the homogeneous ES systems and without consideration of the load profiles. In contrast, a multi-agent cooperative control strategy was proposed to share the loads, based on the frequency differences, among the heterogeneous ES devices [11]. In [11], leader agents and corresponding communication links between the batteries and ultracapacitors were required for the microgrid and ultracapacitors voltages restoration and the batteries and ultracapacitor SoC balancing. In addition, to remove potential single points of failure, multiple leaders were introduced.

Motived by the above discussions, this paper proposes a virtual capacitor based cooperative control for a datacenter DC microgrid with distributed heterogeneous ES devices to coordinate power sharing. Fig. 1 depicts configuration of the datacenter. Each ES device is interfaced to the microgrid via a DC-DC converter. The proposed control architecture has two levels: primary and secondary level. At the primary level, the virtual resistance and capacitance control schemes allocate low and high frequency components of load to batteries and ultracapacitors respectively. At the secondary level, the voltage restoration and $\mathrm{SoC}$ balancing are achieved through a distributed communication network.

Compared with the previous works on the virtual impedance and distributed consensus control, this paper is different and improves on the following aspects. In [28], the virtual impedance control was extended by adding an extra virtual capacitor into the primary droop control for the supercapacitor to absorb surge currents. In this paper, the virtual resistor is replaced by a virtual capacitor for the ultracapacitor systems, while the virtual resistor is still implemented for the battery systems. As a result, the proposed scheme emulates an RC circuit which allows to allocate the high frequency component of load to the ultracapacitors and the low frequency component of load to the batteries. As a result, the controller is simplified and the control parameters are easier to design. Also, only the virtual capacitor value needs to be found and the equation for the time constant is simpler than that of the equation in [28]. In addition, the control system in this paper also restores voltages of the DC microgrid. Furthermore, [29] applied a consensus algorithm for the voltage restoration and current sharing. However, in this paper, the voltage regulation requirement includes the line impedance effect. As a result, the average consensus algorithm regulates the average voltage across the microgrid in response to the global voltage reference. Furthermore, different from the current sharing strategy in [29], this paper proposes SoC balancing strategy to share the low frequency component of loads.

The novel features of the proposed strategy are:

1) The proposed strategy removes the communication link between batteries and ultracapacitors for the allocation of loads, and the communication links between ultracapacitors for the their voltage restoration. 


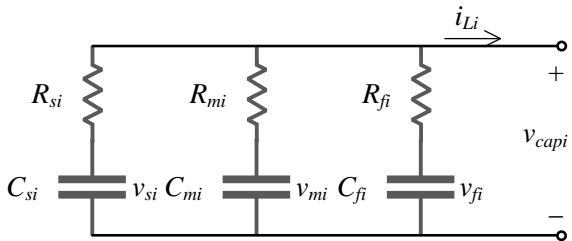

Fig. 2. Ultracapacitor three time constant equivalent circuit model.

2) The batteries and ultracapacitors automatically respond to different components of loads through the primary control, even when there is a communication link failure.

3) The implementation of average consensus removes the requirement for leader agents.

4) The capacitance of ultracapacitors is precisely sized.

5) The coordination controllers and decentralized controllers are simplified, while ensuring the stability.

6) The proposed approach is resilient to a communication link failure and has plug-and-play capability.

7) The proposed control strategy allows integration of renewable energy sources, such as photovoltaic generation, into the DC microgrid.

8) The effects of the secondary controls and the basic converter controls on the virtual capacitance control are analyzed in the design procedure.

The rest of this paper is organized as follows. Section II describes the dynamics of ES devices: batteries and ultracapacitors. Section III explains the principle of operation of the proposed control strategy. Section IV analyses the stability of the microgrid. Section V provides real-time digital simulation results, verifying the performance of the proposed control strategy. Section VI concludes the paper.

\section{EnERgy Storage Systems}

This section provides the models of batteries and ultracapacitors. These functions are used to prove the stability of the global microgrid in Section IV.

\section{A. Battery Modelling}

The first order model of state of charge (SoC) of the $i$-th battery is, based on the SoC definition in [30],

$$
S \dot{S o} C_{i}=-\frac{i_{L i}}{C_{b a t t i}^{\max }},
$$

where $S o C_{i}$ is the battery $\mathrm{SoC}, i_{L i}$ is the inductor current of the DC-DC converter, and $C_{b a t t i}^{\max }$ is the battery charge capacity. At a particular steady state output current, an electrochemical battery cell (lead-acid) can be modeled as an open circuit voltage $v_{o c i}$ and RC network, each with a nonlinear dependence on the SoC,

$$
\begin{aligned}
& v_{o c i}=a_{0 i} e^{-a_{1 i} S o C_{i}}+a_{2 i}+a_{3 i} S o C_{i}-a_{4 i} S o C_{i}^{2}+a_{5 i} S o C_{i}^{3}, \\
& r_{s i}=b_{0 i} e^{-b_{1 i} S o C_{i}}+b_{2 i}+b_{3 i} S o C_{i}-b_{4 i} S o C_{i}^{2}+b_{5 i} S o C_{i}^{3}, \\
& r_{t s i}=c_{0 i} e^{-c_{1 i} S o C_{i}}+c_{2 i}, c_{t s i}=d_{0 i} e^{-d_{1 i} S o C_{i}}+d_{2 i}, \\
& r_{t l i}=e_{0 i} e^{-e_{1 i} S o C_{i}}+e_{2 i}, c_{t l i}=f_{0 i} e^{-f_{1 i} S o C_{i}}+f_{2 i}
\end{aligned}
$$

where $r_{s i}, r_{t s i}, c_{t s i}, r_{t l i}$, and $c_{t l i}$ are non-linear RC network resistors and capacitors. Specifically, the series resistance $r_{s i}$ characterizes the charging/discharging energy losses of the battery. The transient resistances and capacitances $r_{t s i}$ and $c_{t s i}$ represent the short-term transient response characteristics, while the transient resistances and capacitances $r_{t l i}$ and $c_{t l i}$ characterize the long-term transient response characteristics. Parameters $a_{*}, b_{*}, c_{*}, d_{*}, e_{*}$ and $f_{*}$, in which $*$ represents different subscripts, are extracted from the battery curve [30], and are given in Table II.

\section{B. Ultracapacitor Modelling}

The ultracapacitors are modelled by the three time constant equivalent circuit model from [11], shown in Fig. 2. The $i$ th ultracapacitor's voltage dynamics can be described by the following state space model,

$$
\begin{aligned}
& \dot{x}_{\text {capi }}=A_{\text {capi }} x_{c a p i}+B_{\text {capi }} i_{L i}, \\
& v_{i}^{c a p}=C_{c a p i} x_{c a p i}+D_{c a p i} i_{L i}, \\
& x_{c a p i}=\left[\begin{array}{lll}
v_{f i} & v_{m i} & v_{s i}
\end{array}\right]^{T},
\end{aligned}
$$

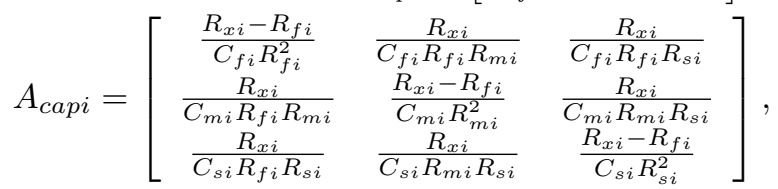

$$
\begin{aligned}
& B_{\text {capi }}=\left[\begin{array}{ccc}
\frac{-R_{x i}}{C_{f i} R_{f i}} & \frac{-R_{x i}}{C_{m i} R_{m i}} & \frac{-R_{x i}}{C_{s i} R_{s i}}
\end{array}\right]^{T}, \\
& C_{\text {capi }}=\left[\begin{array}{lll}
\frac{R_{x i}}{R_{f i}} & \frac{R_{x i}}{R_{m i}} & \frac{R_{x i}}{R_{s i}}
\end{array}\right], D_{c a p i}=\left[-R_{x i}\right], \\
& R_{x i}=\left(1 / R_{f}+1 / R_{m}+1 / R_{s}\right)^{-1},
\end{aligned}
$$

where $v_{i}^{c a p}$ is the ultracapacitor voltage and $i_{L i}$ is the DC-DC converter inductor current.

\section{Bidirectional DC-DC Converter Modeling}

Each ES device connects to the microgrid through a bidirectional DC-DC converter [11], [31], which allows the ES device to charge/discharge. A PI voltage control loop and an inner PI current control loop are implemented for the DC-DC converter. The output voltage of the DC-DC converter $v_{i}$ is given by,

$$
v_{i}=G_{\text {convi }} v_{i}^{r e f},
$$

where $v_{i}^{r e f}$ is the voltage reference signal from the secondary level controller and virtual impedance controller presented in the following section and $G_{c o n v i}$ is the transfer function of the converter and its control system given in [11].

\section{PRinciple of Operation}

Each ES device is connected to the microgrid with a controllable bidirectional DC-DC converter. An ES system incorporates the ES device, DC-DC converter and its control system. The ES systems are linked by a sparse communication network allowing different data exchange between neighbors. Specifically, two types of data are exchanged via the communication network: 1) the average estimation of output voltages and 2) SoC of batteries.

A hierarchical control structure is used for the heterogeneous energy storages. At the primary control level, each ES device uses the voltage loop with an inner current loop control 
strategy [31]. The virtual resistance droop control is introduced to batteries for feeding only the low frequency component of loads, while the virtual capacitive control is implemented for ultracapacitors to supply the high frequency component of loads. At the secondary control level, the average consensus protocol restores the bus voltages of battery systems to the rated values and the cooperative control balances the SoC between batteries. At the same time, the ultracapacitor voltages are restored locally.

Since the virtual impedance control is at the primary level, it has to be faster than the secondary level controls. At the secondary level, the primary objective is to maintain the local bus voltages at the rated value. Therefore, the average consensus control among local bus voltages has to be tuned to be faster than the SoC balancing control and the ultracapacitor voltage restoration control.

\section{A. Virtual Resistance Control for Battery Systems}

In the diagram of the battery control system, the voltage reference in (4) for the DC-DC converter is set by the V-I droop control modified with two correction terms, generated by the secondary controllers. The proportional load sharing objective of droop control is replaced by the SoC balancing in this paper and provided by the secondary SoC cooperative control. Therefore, the objective of the V-I droop control is to allocate different components of loads by two types of droop control: virtual resistance control and virtual capacitive control [17]. The virtual resistance based droop control enables the battery to only provide the low frequency component of loads. While the virtual capacitive control allows the ultracapacitors to feed the high frequency component of loads. The voltage reference signal for the DC-DC converter of the battery system is

$$
v_{i}^{r e f}=v_{m g}-r_{i}^{d r p} i_{i}+\delta v_{1 i}+\delta v_{2 i}^{b},
$$

where $v_{m g}$ is the rated voltage of the microgrid (assumed to be a constant value), $r_{i}^{d r p}$ is the virtual resistor, $i_{i}$ is the output current of the converter, $\delta v_{1 i}$ is the bus voltage correction term and $\delta v_{2 i}^{b}$ is the SoC balancing correction term. The parameter $r_{i}^{d r p}$ is tuned using method in [17] as

$$
r_{i}^{d r p}=\frac{\Delta v}{P_{\max } / v_{\min }},
$$

where $\Delta v$ is the maximum allowed deviation of the microgrid voltage, $P_{\max }$ is the maximum output power from the converter and $v_{\min }$ is the minimum allowed microgrid voltage.

\section{B. Virtual Capacitive Control for Ultracapacitor Systems}

Similar to (5), in the virtual capacitive control for the ultracapacitors, the virtual resistor is replaced by a capacitor,

$$
v_{i}^{r e f}=v_{m g}-\frac{1}{c_{i}^{d r p} s} i_{i}+\delta v_{i}^{c},
$$

where $c_{i}^{d r p}$ is the virtual capacitor and $\delta v_{2 i}^{c}$ is the ultracapacitor voltage correction term. The virtual capacitance is determined from the virtual resistance and the corner frequency $\omega_{i}$ [17],

$$
\omega_{i}=1 /\left(r_{i}^{d r p} c_{i}^{d r p}\right)
$$

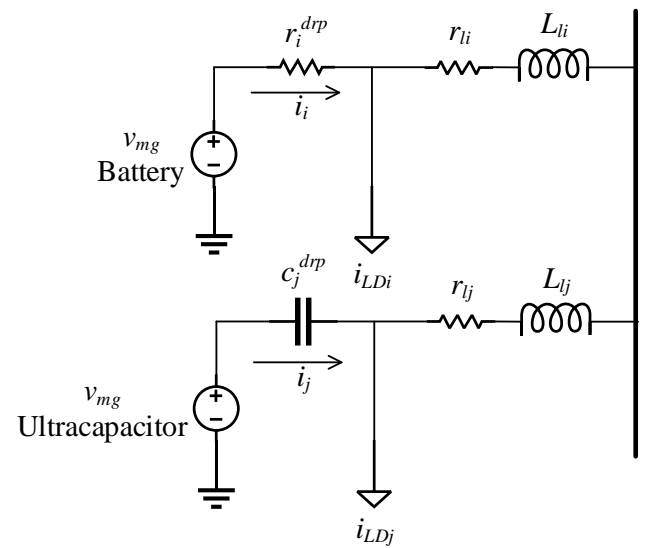

Fig. 3. A simplified microgrid with a battery, an ultracapacitor and two distributed loads, under virtual capacitance control.

\section{Effects of Virtual Capacitance Control}

To illustrate the effects of the virtual capacitance control, a simple microgrid with a battery and an ultracapacitor, as shown in Fig. 3, is considered. From Fig. 3 the battery and ultracapacitor systems currents $i_{i}$ and $i_{j}$ can be obtained as

$$
\begin{aligned}
i_{i}= & \frac{c_{j}^{d r p}\left(L_{l i}+L_{l j}\right) s^{2}+c_{j}^{d r p}\left(r_{l i}+r_{l j}\right) s+1}{c_{j}^{d r p}\left(L_{l i}+L_{l j}\right) s^{2}+c_{j}^{d r p}\left(r_{l i}+r_{l j}+r_{i}^{d r p}\right) s+1} i_{L D i} \\
& +\frac{1}{c_{j}^{d r p}\left(L_{l i}+L_{l j}\right) s^{2}+c_{j}^{d r p}\left(r_{l i}+r_{l j}+r_{i}^{d r p}\right) s+1} i_{L D j}, \\
i_{j}= & \frac{c_{j}^{d r p} r_{i}^{d r p} s}{c_{j}^{d r p}\left(L_{l i}+L_{l j}\right) s^{2}+c_{j}^{d r p}\left(r_{l i}+r_{l j}+r_{i}^{d r p}\right) s+1} i_{L D i} \\
& +\frac{c_{j}^{d r p}\left(r_{l i}+r_{l j}+r_{i}^{d r p}\right) s+c_{j}^{d r p}\left(L_{l i}+L_{l j}\right) s^{2}}{c_{j}^{d r p}\left(L_{l i}+L_{l j}\right) s^{2}+c_{j}^{d r p}\left(r_{l i}+r_{l j}+r_{i}^{d r p}\right) s+1} i_{L D j},
\end{aligned}
$$

where $r_{l i, j}$ and $L_{l i, j}$ represent the line resistance and inductance respectively. Since the virtual capacitance and resistance values are much larger than the line impedances, ignoring the line impedances in (9) gives

$$
\begin{aligned}
i_{i} & =\frac{1}{c_{j}^{d r p} r_{i}^{d r p} s+1}\left(i_{L D i}+i_{L D j}\right), \\
i_{j} & =\frac{c_{j}^{d r p} r_{i}^{d r p} s}{c_{j}^{d r p} r_{i}^{d r p} s+1}\left(i_{L D i}+i_{L D j}\right) .
\end{aligned}
$$

From (10), it is obvious that there is a low pass filtering effect on $\frac{i_{i}}{i_{L D i}}$ and $\frac{i_{i}}{i_{L D j}}$, and a high pass filtering effect on $\frac{i_{j}}{i_{L D i}}$ and $\frac{i_{j}}{i_{L D j}}$, with the corner frequency $\frac{1}{c_{j}^{d r p} r_{i}^{d r p}}$.

The step responses of the power system are shown in Fig. 4. The ultracapacitor immediately supplies the load and then decreases its output power to zero. Meanwhile, the battery gradually increases its output power and supports the load during the steady state.

Fig. 5 shows the step responses without considering the RL lines impedances $\left(r_{l i, j}=0, L_{l i, j}=0\right)$ in Fig. 3. Comparing Fig. 4 and Fig. 5, it can be observed in Fig. 4 that the ultracapacitor cannot fully supply the initial current at $t=0$. However, without considering the RL lines impedances, the ultracapacitor fully supplies the initial current, as shown in Fig. 5. This is because the RL line impedances affect the transfer functions (9).

Remark 1: The virtual capacitance control replaces the virtual resistor by a virtual capacitor for ultracapacitor systems. Similar to the virtual resistor, the virtual capacitor would not cost or restore any power. As a result, in terms of energy, 


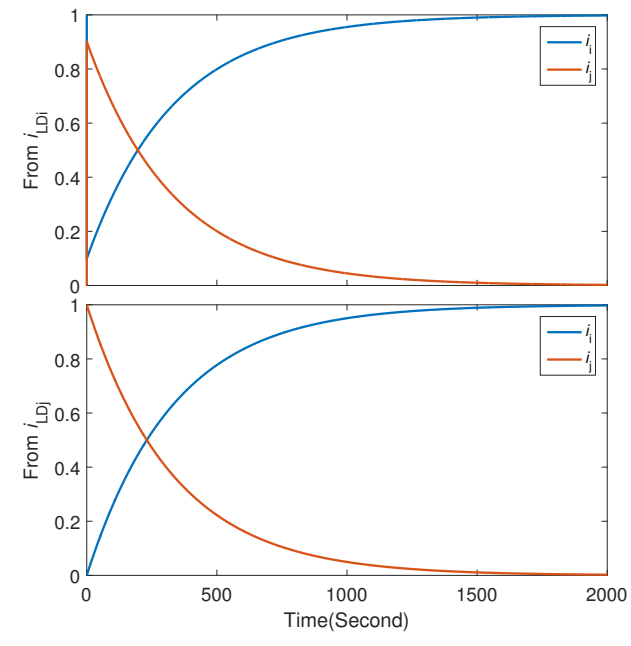

Fig. 4. Responses of currents $i_{i}$ and $i_{j}$ to a step change in the loads $i_{L D i}$ and $i_{L D j}$ (one at a time), for the simplified microgrid shown in Fig. 3.

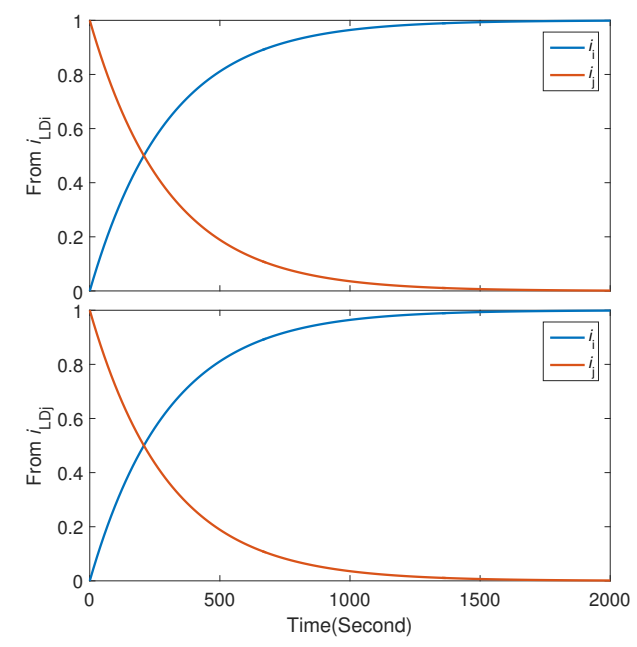

Fig. 5. Responses of currents $i_{i}$ and $i_{j}$ to a step change in the loads $i_{L D i}$ and $i_{L D j}$ (one at a time), for the simplified microgrid shown in Fig. 3 and without considering the RL lines $\left(r_{l i, j}=0, L_{l i, j}=0\right)$.

the virtual capacitor will not result in an effectively lower capacitance of the ultracapacitor.

Remark 2: The charge and discharge rates of the ultracapacitors are controlled by the virtual capacitance controller at short timescale, while the ultracapacitor voltage restoration at long timescale. The virtual capacitance control provides power sharing between ultracapacitors, similar to that of the virtual resistor droop control among batteries. Furthermore, the slow ultracapacitor voltage restoration control maintains the ultracapacitor voltage at the nominal value to ensure that the ultracapacitors will have sufficient energy for any upcoming fast variation in loads/sources.

To illustrate the power sharing between ultracapacitors, a simple microgrid, shown in Fig. 6, with a battery and two ultracapacitors is modeled. The output current of each energy storage agent can be expressed as

$$
i=\Phi i_{L D},
$$

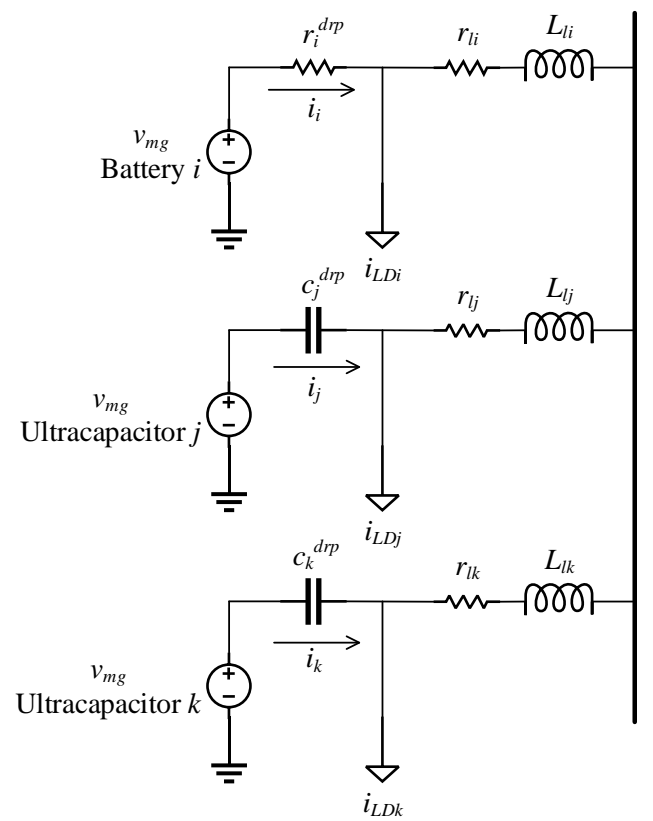

Fig. 6. A simple microgrid with a battery and two ultracapacitors to illustrate the discharge/charge rate control between ultracapacitors.

where

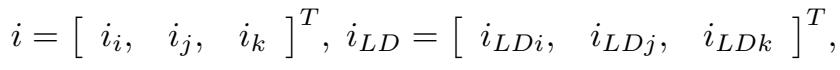

represent the energy storage output currents and the distributed load currents respectively, and $\Phi$ is given by (12). The step responses in Fig. 7 show that the ultracapacitors can immediately proportionally share the high frequency component of the step change in load. Now, assume that the corner frequency for the ultracapacitor $k$ is set to ten times of the $j$. Then, the step responses shown in Fig. 8 illustrate that the ultracapacitor $k$ will supply less power than the ultracapacitor $j$ during the transient stage.

\section{Sizing of Ultracapacitors}

The required equivalent capacitance of the ultracapacitors can be calculated based on the corner frequency of the virtual capacitive control and a specific load/power profile as [14],

$$
C_{u c}^{e q}=\frac{4 \max \left(\left|d E_{u c}\right|\right)}{v_{u c, \max }^{2}-v_{u c, \min }^{2}}, d E_{u c}=G_{u c} P_{m g} / s,
$$

where $C_{u c}^{e q}$ is equivalent capacitance of the ultracapacitors distributed in a microgrid, $d E_{u c}$ is the ultracapacitor energy change, $v_{u c, \max }$ and $v_{u c \text {, min }}$ are the maximum and minimum ultracapacitor voltage limits, $G_{u c}=s / \omega$ is the transfer function between the ultracapacitor output power and the heterogeneous ES system output power based on virtual capacitive control, $P_{m g}$ is the load/power profile in the microgrid.

\section{E. Average Consensus for Bus Voltages Restoration}

The DC microgrid ES system are connected by a sparse communication graph $\mathcal{G}(\mathcal{V}, \mathcal{E})$, with nodes $\mathcal{V}=\{1, \cdots, N\}$ and edges $\mathcal{E}$ [32]. Each graph node represents an ES system and the graph edges represent communication links between 


$$
\begin{aligned}
& \Phi=\left[\begin{array}{ccc}
\frac{\left(Z_{k}^{d r p}+Z_{k i}\right)\left(Z_{j}^{d r p}+Z_{i j}\right)}{\Xi_{i}} & \frac{Z_{j}^{d r p}\left(Z_{k}^{d r p}+Z_{j k}\right)}{\Xi_{j}} & \frac{Z_{k}^{d r p}\left(Z_{j}^{d r p}+Z_{j k}\right)}{\Xi_{k}} \\
\frac{Z_{i}^{d r p}\left(Z_{k}^{d r p}+Z_{k i}\right)}{\Xi_{i}} & \frac{\left(Z_{i}^{d r p}+Z_{i j}\right)\left(Z_{k}^{d r p}+Z_{j k}\right)}{\Xi_{j}} & \frac{Z_{k}^{d r p}\left(Z_{i}^{d r p}+Z_{k i}\right)}{\Xi_{k}} \\
\frac{Z_{i}^{d r p}\left(Z_{j}^{d r p}+Z_{i j}\right)}{\Xi_{i}} & \frac{Z_{j}^{d r p}\left(Z_{i}^{d r p}+Z_{i j}\right)}{\Xi_{j}} & \frac{\left(Z_{i}^{d r p}+Z_{k i}\right)\left(Z_{j}^{d r p}+Z_{j k}\right)}{\Xi_{k}}
\end{array}\right], \\
& \Xi_{i}=\left(Z_{k}^{d r p}+Z_{k i}\right)\left(Z_{j}^{d r p}+Z_{i j}\right)+Z_{i}^{d r p}\left(Z_{j}^{d r p}+Z_{i j}\right)+Z_{i}^{d r p}\left(Z_{k}^{d r p}+Z_{k i}\right), \\
& \Xi_{j}=\left(Z_{i}^{d r p}+Z_{i j}\right)\left(Z_{k}^{d r p}+Z_{j k}\right)+Z_{j}^{d r p}\left(Z_{k}^{d r p}+Z_{j k}\right)+Z_{j}^{d r p}\left(Z_{i}^{d r p}+Z_{i j}\right), \\
& \Xi_{k}=\left(Z_{i}^{d r p}+Z_{k i}\right)\left(Z_{j}^{d r p}+Z_{j k}\right)+Z_{k}^{d r p}\left(Z_{j}^{d r p}+Z_{j k}\right)+Z_{k}^{d r p}\left(Z_{i}^{d r p}+Z_{k i}\right), \\
& Z_{i}^{d r p}=r_{i}^{d r p}, Z_{j, k}^{d r p}=\frac{1}{c_{j, k}^{d r p} s}, Z_{i j}=Z_{i}+Z_{j}, i, j=i, j, k, Z_{i}=r_{l i}+L_{l i} s, i=i, j, k .
\end{aligned}
$$

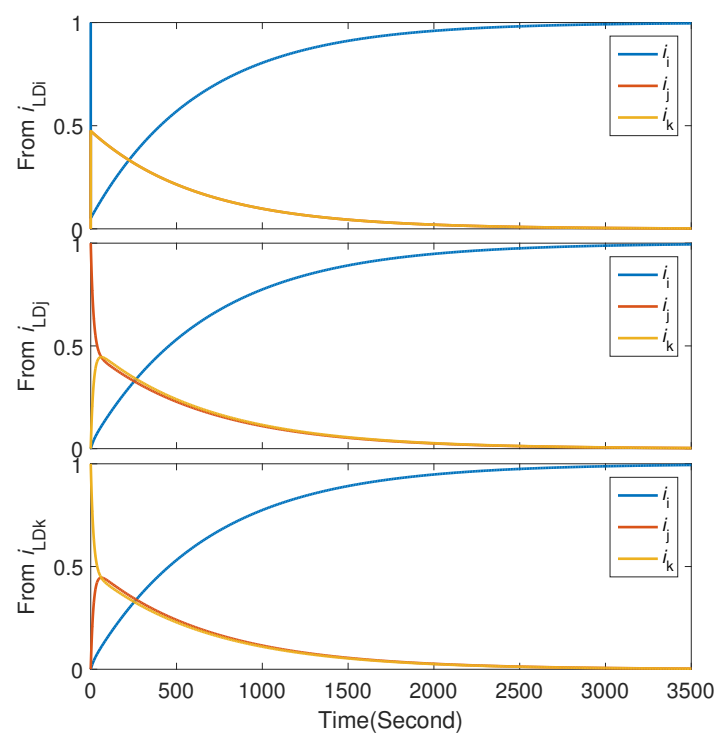

Fig. 7. Responses of currents $i_{i}, i_{j}$ and $i_{k}$ to a step change in the loads $i_{L D i}, i_{L D j}$ and $i_{L D k}$ (one at a time), for the simplified microgrid shown in Fig. 6 and with equal virtual capacitance.

them. $(i, j) \in \mathcal{E}$ if there is a link allowing information to flow from node $i$ to node $j$. The neighbors of node $i$ are given by $\mathcal{N}_{i}$, where $j \in \mathcal{N}_{i}$, if $(i, j) \in \mathcal{E}$. The graph adjacency matrix is given by

$$
\mathcal{A}=\left[a_{i j}\right] \in \mathbb{R}^{N \times N}, a_{i j}=\left\{\begin{array}{l}
1,(j, i) \in \mathcal{E} \\
0, \text { otherwise }
\end{array} .\right.
$$

The graph Laplacian matrix is given by $L=\mathcal{D}-\mathcal{A}$, where $\mathcal{D}=\operatorname{diag}\left\{d_{i}\right\}$, and $d_{i}=\sum_{j=1}^{N} a_{i j}$ is the in-degree of the communication network. The graph $\mathcal{G}$ describes only the communication network between battery ES systems. The graph is bidirectional, which allows each battery ES system to receive/send information from/to neighbors.

Through the communication links, the local observer estimates the average values of state variables based on the information from its neighbors $j \in \mathcal{N}_{i}$. The following distributed average consensus protocol [5] is implemented.

$$
\bar{z}_{i}=z_{i}+\int \sum_{j \in \mathcal{N}_{i}} a_{i j}\left(\bar{z}_{j}-\bar{z}_{i}\right) d t
$$

where $z_{i}$ is a local state variable, $\bar{z}_{i}$ is the local estimation of the average value of the state based on neighbors information.

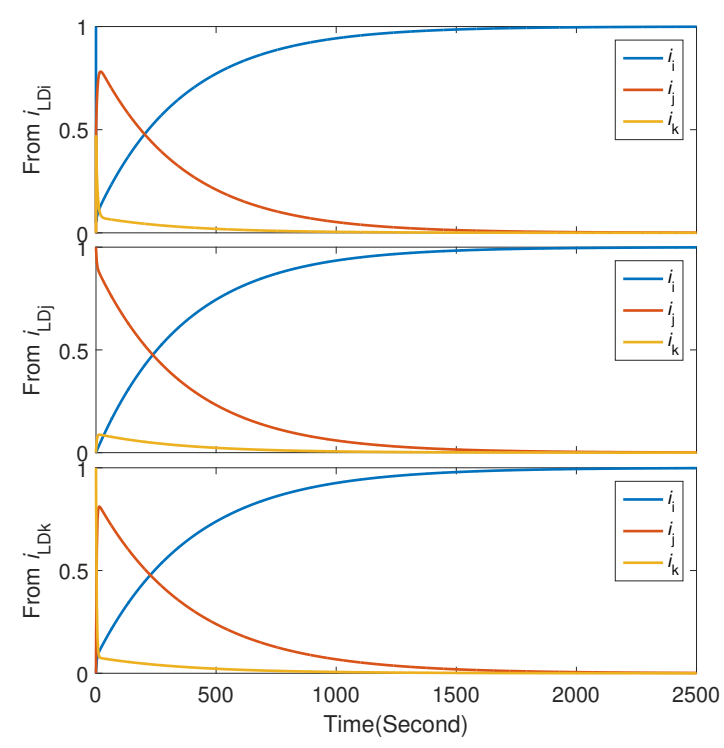

Fig. 8. Responses of currents $i_{i}, i_{j}$ and $i_{k}$ to a step change in the loads $i_{L D i}, i_{L D j}$ and $i_{L D k}$ (one at a time), for the simplified microgrid shown in Fig. 6 and with unequal virtual capacitance.

Assume that the microgrid contains $N$ ES systems, including $p$ battery systems and $q$ ultracapacitor systems $(N=p+q)$. In accordance with (15), the average consensus protocol of each battery local bus voltage through the graph $\mathcal{G}$ gives

$$
\bar{v}_{i}=v_{i}+\int \sum_{j \in \mathcal{N}_{i}} a_{i j}\left(\bar{v}_{j}-\bar{v}_{i}\right) d t
$$

where $\bar{v}_{i}$ is the estimation of the local bus voltage. Therefore, the $\left\{\bar{v}_{i}\right\}$ are exchanged through communication network between batteries for local bus voltage average consensus protocol.

In order to maintain the average battery local bus voltage at the rated value $v_{m g}$, a PI controller is required [33]. Then, the local bus voltage correction term in (5) is given by

$$
\delta v_{1 i}=H_{i}\left(v_{m g}-\bar{v}_{i}\right), H_{i}=k_{\bar{v} P i}+\frac{k_{\bar{v} I i}}{s},
$$

where $H_{i}$ is the PI controller, $k_{\bar{v} P i}$ and $k_{\bar{v} I i}$ are proportional and integral gains respectively. This average voltage controller regulates the average value of the local bus voltages of the battery system to the rated microgrid voltage. Thus, the bus 


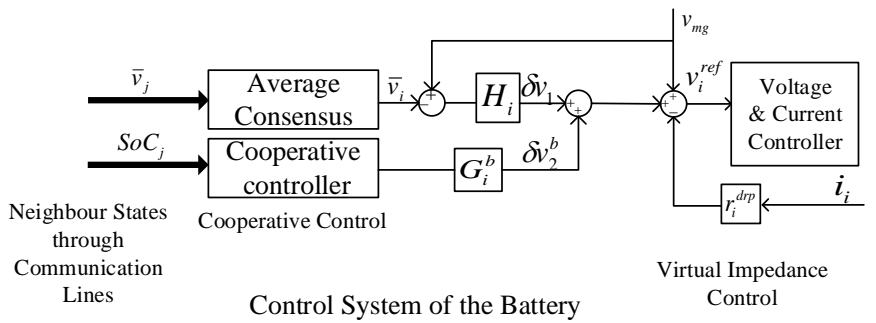

Fig. 9. The control system for the battery DC-DC converter.

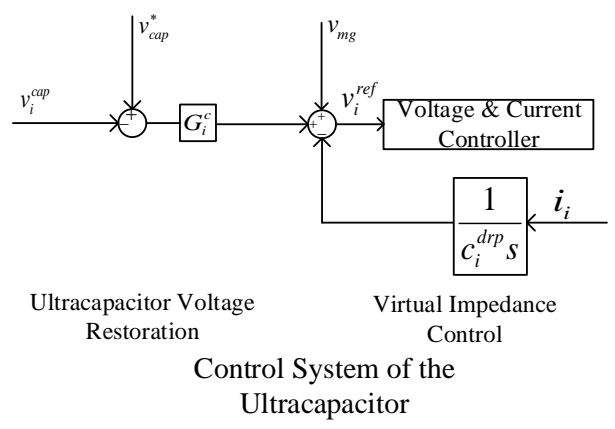

Fig. 10. The control system for the ultracapacitor DC-DC converter.

voltage divergence, resulted from the primary droop control, is mitigated.

Remark 3: The line impedance causes a trade-off between the accurate power sharing and voltage regulation. By restoring the average value of the voltage to the rated value the line impedance effects can be compensated and accurate power sharing between batteries ( $\mathrm{SoC}$ balancing) can be achieved.

\section{F. Cooperative Control for Battery Systems SoC Balancing}

A cooperative control is introduced to balance the SoC between batteries. The data of $\left\{S o C_{i}\right\}$ are exchanged between neighboring batteries. The correction term $\delta v_{2 i}^{b}$ in (5) is defined as

$$
\delta v_{2 i}^{b}=G_{i}^{b} \sum_{j \in \mathcal{N}_{i}} a_{i j}\left(S o C_{j}-S o C_{i}\right), G_{i}^{b}=k_{b P i},
$$

where $k_{b P i}$ is the proportional control gain of the cooperative control for SoC balancing.

\section{G. Ultracapacitor Voltages Restoration}

A local control method for the regulation of ultracapacitor voltage ensures the voltage is maintained at the rated value to allow feeding power peaks. Since this controller restores the ultracapacitor voltage, it also helps to eliminate the ultracapacitors' voltage divergence caused by the virtual capacitive control [16]. A local PI controller sets the ultracapacitor voltage correction term in (7) as

$$
\delta v_{i}^{c}=G_{i}^{c}\left(v_{c a p}^{*}-v_{i}^{c a p}\right), G_{i}^{c}=k_{c P i}+\frac{k_{c I i}}{s},
$$

where $v_{c a p}^{*}$ is the rated value of ultracapacitor voltage, $k_{c P i}$ and $k_{c I i}$ are the proportional and integral gains respectively.

Figs. 9 and 10 show the block diagrams of the proposed control strategy for a battery and an ultracapacitor. There

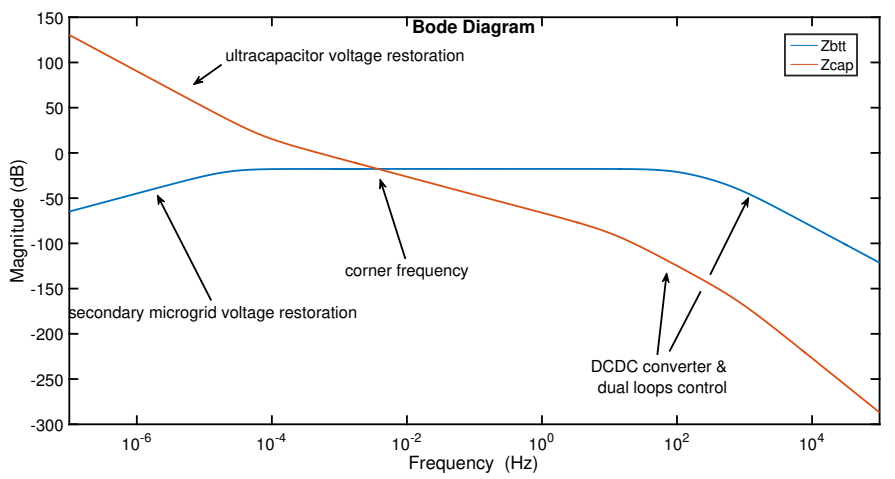

Fig. 11. Bode plots of the battery system (Zbtt) and the ultracapacitor system (Zcap), used for tuning of the DC-DC converter control loops, primary control and secondary control.

is no physical connection between the two control systems and hence no communication between the battery systems and ultracapacitor systems is required. This is one of the main advantages/contributions of the paper. The frequency response of the two control systems is determined by the corner frequency (10), as explained in Section III. C.

Remark 4: The datacenter microgrid in this paper is able to operate in both the grid connected and islanded mode. The differences between two operation modes are: in the islanded mode, the global average voltage reference equals to the rated voltage of the microgrid; in the grid connected mode, the reference is provided by the upper network for the power exchange between the microgrid and main grid [5].

\section{H. Output Impedances}

To illustrate the relationships between the DC-DC converter control loops, primary control and secondary control, the output impedances of the battery system and ultracapacitor system are defined as $Z_{b t t}$ and $Z_{c a p}$ respectively,

$$
\begin{gathered}
Z_{b t t}=\frac{-r_{d r p} G_{c o n v}}{1+K_{v 2 p i} G_{c o n v}}, K_{v 2 p i}=k_{\bar{v} P}+\frac{k_{\bar{v} I}}{s} \\
Z_{c a p}=\frac{v_{m g} K_{c v p i}-v_{c a p}^{*} C_{u c} s}{v_{c a p}^{*} C_{u c} c_{d r p} s^{2}} G_{c o n v}, K_{c p i}=k_{c P}+\frac{k_{c I}}{s} .
\end{gathered}
$$

The Bode plots of $Z_{b t t}$ and $Z_{c a p}$ for Case A are shown in Fig. 11. The DC-DC converter dual voltage and current control loops and the secondary voltage control have to leave sufficient space for the primary control. Hence, the DC-DC converter outer voltage and inner current control loops have to be tuned to be faster than the corner frequency. On the other hand, the secondary level voltage restorations controls have to be tuned slower than the corner frequency. This will ensure proper load allocation by the primary virtual capacitance control.

\section{Design Procedure}

The following steps summarize the proposed method design procedure.

1) Design the primary droop control and virtual capacitance control using (5) and (6), where the corner frequency 
determines the separation of the high and low frequency components of load.

2) Design the outer voltage control and inner current control loops for the DC-DC converter. From the Bode plot in Fig. 11, the overall DC-DC converter response has to be tuned to be at least one order faster than the corner frequency.

3) Size ultracapacitors using (9) in Section III. D.

4) The communication network for batteries requires bidirectional channels and must contain a spanning tree. The value of the communication weights determines the convergence speed of the average voltage and balanced SoC levels. The communication graph must satisfy Lemma 1 in APPENDIX.

5) Design the distributed voltage restoration and SoC balancing secondary control level to be at least one order slower than the primary control level and the inner current and outer voltage loops for the DC-DC converter. The control parameters must meet the stability analysis provided in APPENDIX.

Remark 5 (the extension/variation of a microgrid): When the characteristics of loads/renewable energy sources change, the corner frequency should be redesigned for proper allocation of loads to specific energy storages. In this case, if the previous ultracapacitor is adequate to supply the high frequency component of loads, the step 3 in the design procedure can be skipped.

\section{Stability ANALYSis}

\section{A. Global Dynamic Model}

The global dynamic model of the controlled battery systems in the microgrid can be obtained based on (1), (4), (5), (17), and (18) as

$$
\begin{aligned}
\mathbf{v}=\left(\mathbf{G}_{c o n v}^{-1}+\left(\mathbf{G} L \mathbf{M}+\mathbf{r}_{d r p}\right) Y_{n e t}+\mathbf{H} \mathbf{H}_{a v g}\right)^{-1} \\
\left(\left(\mathbf{H}+I_{N}\right) \mathbf{v}_{m g}\right) .
\end{aligned}
$$

where $\mathbf{H}_{a v g}$ is the transfer function of the battery local bus voltage; $\mathbf{v}_{m g}$ is the rated microgrid voltage, $Y_{n e t}$ is the microgrid bus admittance matrix, $I_{N}$ is an $N \times N$ identity matrix, $v_{b a t t}^{*}$ is the rated battery voltage.

$$
\begin{aligned}
& \mathbf{v}=\left[v_{1}, v_{2}, \cdots, v_{p}\right]^{T}, \mathbf{r}_{d r p}=\operatorname{diag}\left\{r_{i}^{d r p}\right\}, \\
& \mathbf{H}=\operatorname{diag}\left\{H_{i}\right\}, \mathbf{G}_{c o n v}=\operatorname{diag}\left\{G_{\text {convi }}\right\}, \\
& \mathbf{G}=\operatorname{diag}\left\{G_{i}^{b}\right\}, \mathbf{M}=\operatorname{diag}\left\{v_{m g} /\left(v_{\text {batt }}^{*} C_{\text {batti }}^{\max } s\right)\right\} .
\end{aligned}
$$

\section{B. Stability Analysis}

Stability analysis ensures that the proposed control method satisfies the operational requirements of the global voltage regulation, SoC balancing and the ultracapacitor voltage restoration. Generally, assume the microgrid voltage reference dynamics as

$$
\mathbf{v}_{m g}=\left(v_{m g} / s\right) \mathbf{1}_{N},
$$

where $\mathbf{1}_{N} \in \mathbb{R}^{N \times 1}$ is the vector whose elements all equal one. The final value theorem is used to analyze the steady states of
TABLE I

CASe Study Parameters

\begin{tabular}{llllll}
\hline$v_{m g}$ & $380 \mathrm{~V}$ & $v_{b a t t}^{*}$ & $208 \mathrm{~V}$ & $v_{c a p}^{*}$ & $176 \mathrm{~V}$ \\
$C_{b a t t i}^{\text {max }}$ & $192 \mathrm{Ah}$ & $E_{b a t t i}$ & $40 \mathrm{kWh}$ & $C_{u c i}$ & $330.0571 \mathrm{~F}$ \\
$r_{i}^{d r p}$ & $0.912 \Omega$ & $\omega_{i}$ & $(1 / 300) \mathrm{rad} / \mathrm{s}$ & $c_{i}^{d r p}$ & $328.9474 \mathrm{~F}$ \\
$f_{d c d c}$ & $2 \mathrm{kHz}$ & $L_{d c d c}$ & $5 \mathrm{mH}$ & $C_{d c d c}$ & $6.8 \mathrm{mF}$ \\
$k_{\bar{v} P i}$ & 6 & $k_{\bar{v} I i}$ & $1 \times 10^{-3}$ & $k_{b P i}$ & -300 \\
$k_{c P i}$ & $1 \times 10^{-5}$ & $k_{c I i}$ & $1 \times 10^{-4}$ & $R_{f i}$ & $2.3 \times 10^{-3} \Omega$ \\
$R_{m i}$ & $2.9824 \Omega$ & $R_{s i}$ & $8.1122 \Omega$ & $C_{f i}$ & $248.8208 \mathrm{~F}$ \\
$C_{m i}$ & $22.4393 \mathrm{~F}$ & $C_{s i}$ & $58.7399 \mathrm{~F}$ & $P_{m g}$ & $28.88 \mathrm{~kW}$ \\
$v_{u c, \max }$ & $190 \mathrm{~V}$ & $v_{u c, \min }$ & $160 \mathrm{~V}$ & $P_{\text {rated }}^{\text {conv }}$ & $10.4 \mathrm{~kW}$ \\
\hline
\end{tabular}

TABLE II

BATTERY MODEL PARAMETERS

\begin{tabular}{llllllllll}
\hline$a_{0 i}$ & -5.429 & $a_{1 i}$ & 117.5 & $a_{2 i}$ & 11.32 & $a_{3 i}$ & 2.706 & $a_{4 i}$ & -2.04 \\
$a_{5 i}$ & 1.026 & $b_{0 i}$ & 1.578 & $b_{1 i}$ & 8.527 & $b_{2 i}$ & 0.7808 & $b_{3 i}$ & -1.887 \\
$b_{4 i}$ & 2.404 & $b_{5 i}$ & -0.649 & $c_{0 i}$ & 2.771 & $c_{1 i}$ & 9.079 & $c_{2 i}$ & 0.22 \\
$d_{0 i}$ & -2423 & $d_{2 i}$ & 75.14 & $e_{0 i}$ & 55 & $e_{1 i}$ & 2.771 & $e_{2 i}$ & 9.079 \\
$f_{0 i}$ & -1240 & $f_{1 i}$ & 9.571 & $f_{2 i}$ & 3100 & & & & \\
\hline
\end{tabular}

the global microgrid dynamics. Assume the steady state global bus voltage vector, $\mathbf{v}^{s s}$,

$$
\begin{gathered}
\mathbf{v}^{s s}=\lim _{s \rightarrow 0}\left(s \mathbf{G}_{c o n v}^{-1}+s\left(\mathbf{G} L \mathbf{M}+\mathbf{r}_{d r p}\right) Y_{n e t}+s \mathbf{H} \mathbf{H}_{a v g}\right)^{-1} \\
\left(\left(s \mathbf{H}+s I_{N}\right) \mathbf{v}_{m g}\right) .
\end{gathered}
$$

Based on the principles from [5], [33], the following results can be obtained from (24),

$$
\left\langle\mathbf{v}^{s s}\right\rangle=v_{m g}, \text { SoC }^{s s}=\varsigma \mathbf{1}_{p},
$$

where $\varsigma$ is a positive scalar, $\mathbf{1}_{p} \in \mathbb{R}^{p \times 1}$ is the vector whose elements all equal one, $\langle\cdot\rangle$ represents the average of all elements in the vector, $\mathbf{S o C}^{s s}$ is the final values of the SoC vector. (25) indicates that the average output voltage of batteries equals to the rated microgrid voltage and the SoCs can converge to the same level.

In addition, the virtual capacitive control [17] and local ultracapacitor voltage restoration control [16] ensure the stability of the ultracapacitor systems.

\section{Results}

Simulations for a $380 \mathrm{~V}$ islanded DC datacenter microgrid [11] were carried out on RTDS Technologies real-time digital simulator, with switching converter models to verify the performance of the proposed control strategy.

The configuration of the datacenter microgrid, with four batteries and ten ultracapacitors, is shown in Fig. 12. Based on the ETSI EN 300 132-3-1 standard, the datacenter microgrid voltage limits are set to $380 \mathrm{~V} \pm 5 \%(360 \mathrm{~V}$ to $400 \mathrm{~V})$ [11]. Each ES device is connected to a local load. Each local bus is connected through RL lines. The parameters of the case studies are presented in Table I and the battery model parameters are given in Table II. Fig. 12 also shows the sparse communication network allowing neighbor-to-neighbor data exchange between battery systems. Two sets of data are exchanged through the communication network: $\left\{S o C_{i}, \bar{v}_{i}\right\}$. 


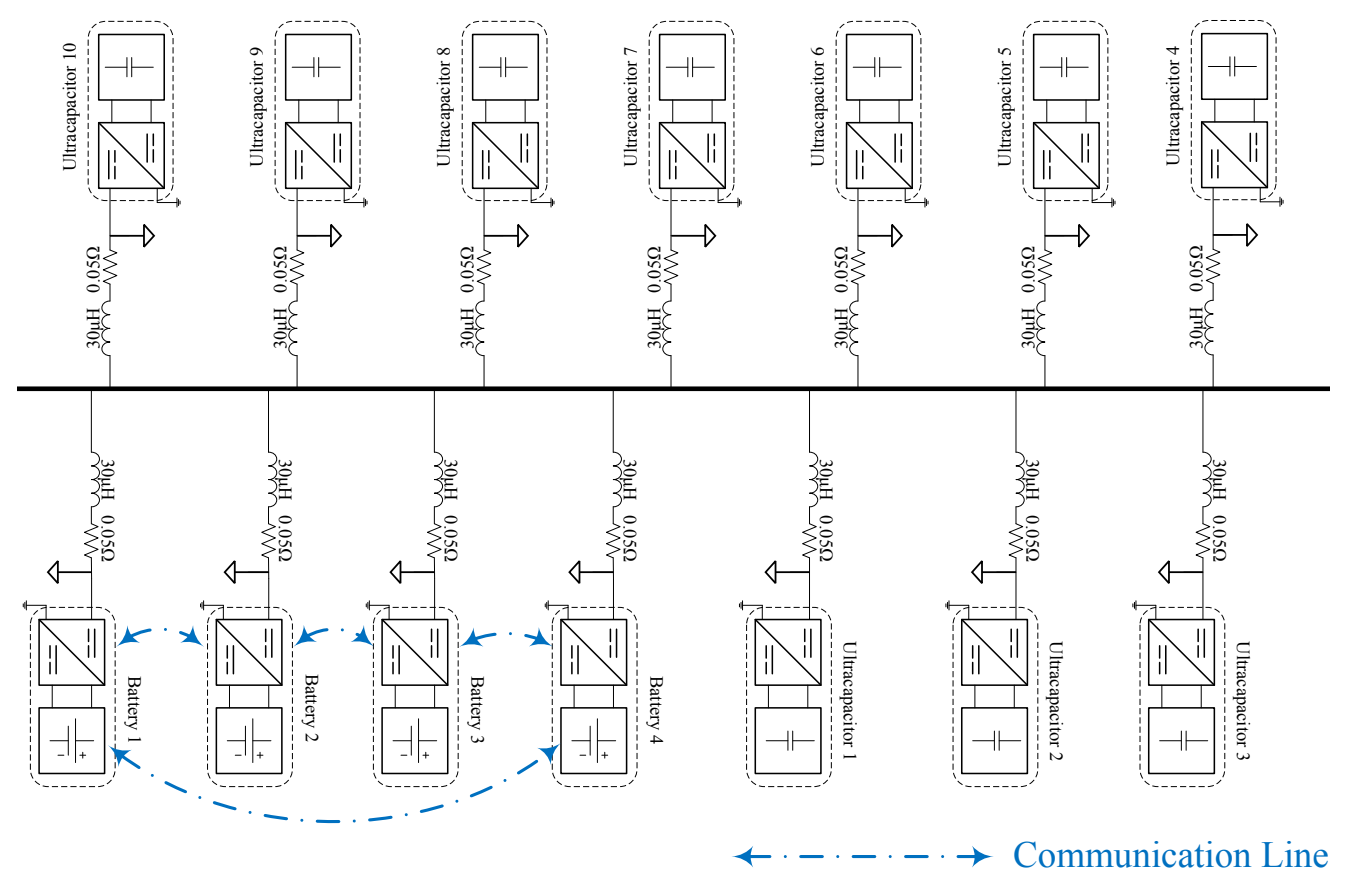

Fig. 12. 380V DC datacenter microgrid.
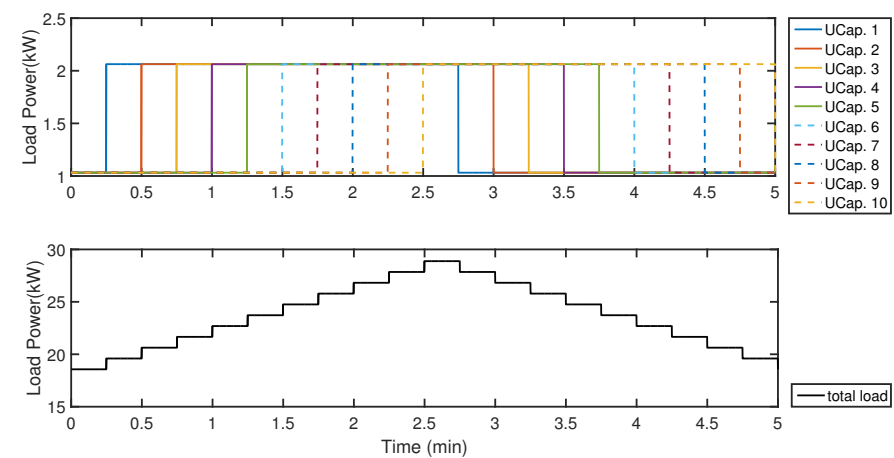

Fig. 13. Case Study A: DC microgrid load profiles over $5 \mathrm{~min}$.

The initial energy levels of battery systems are between $70 \%$ and $100 \%$. The capacitance of ultracapacitors is found from (13) and the voltage limits are $160 \mathrm{~V} \leqslant v_{i}^{c a p} \leqslant 190 \mathrm{~V}$.

Three case studies are presented. Case Study A verifies performance of the proposed strategy on the datacenter with variable loads. Case Study B verifies resiliency to a single link failure and plug-and-play capability. Case Study C presents the application of the proposed control method to a microgrid with PV sources.

\section{A. Case Study A. Variable Loads}

In this case study, the datacenter operates in the islanded mode. Each battery system has a $40 \mathrm{kWh}$ lead-acid battery and $70 \Omega(2.0629 \mathrm{~kW}$ at $380 \mathrm{~V})$ constant load. Each ultracapacitor has a $140 \Omega(1.0315 \mathrm{~kW}$ at $380 \mathrm{~V})$ constant load and $140 \Omega$ $(1.0315 \mathrm{~kW}$ at $380 \mathrm{~V})$ variable load, which switches on for $2.5 \mathrm{~min}$ at every $5 \mathrm{~min}$. The variable load is based on statistical analysis of datacenter load magnitudes and peak/valley durations [11]. A 5 minute duration of the periodic load profiles is

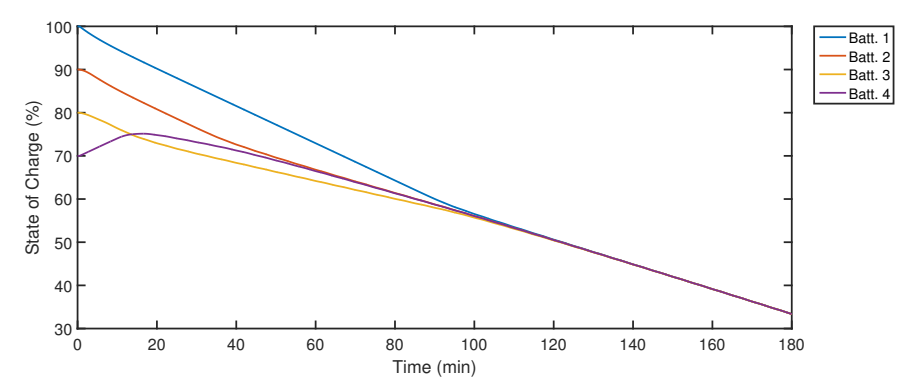

Fig. 14. Case Study A: Battery SoC levels.

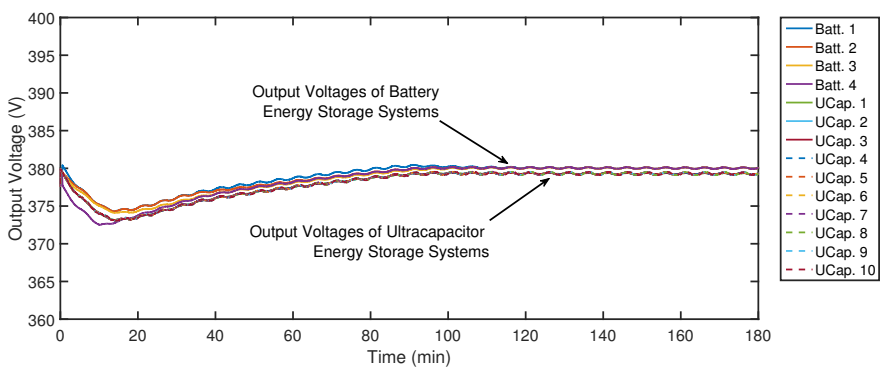

Fig. 15. Case Study A: Local bus voltages.

shown in Fig. 13. The overall load varies between $18.567 \mathrm{~kW}$ and $28.880 \mathrm{~kW}$ with the $5 \mathrm{~min}$ period.

In Figs. 14-16, initially, the ultracapacitors supply all loads distributed in the datacenter microgrid due to initial step change in the load profile, which is treated as the high frequency component of loads. Subsequently, the batteries supply the steady loads as well as energy to the ultracapacitors on a slower time-scale, restoring the ultracapacitor voltages to the rated value. The SoC of batteries reach same level at $80 \mathrm{~min}$. On the faster time-scale, the ultracapacitors continue 


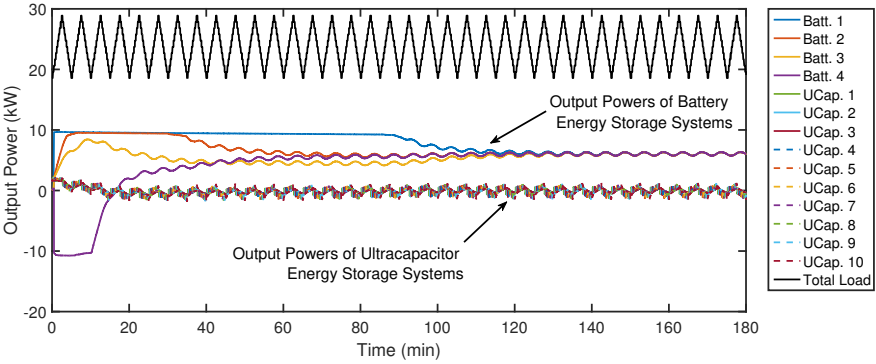

Fig. 16. Case Study A: ES device output powers.

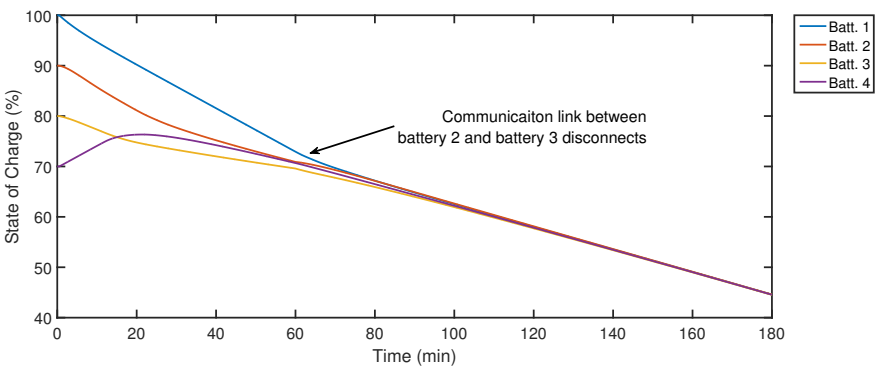

Fig. 17. Case Study B: 1) Battery SoC levels.

supplying the variable peak/valley components of the loads.

The battery DC-DC converters are rated at $10.4 \mathrm{~kW}$. As a result, the batteries avoid overloading/overcharging during the transient stage of SoC balancing, as shown in Fig. 16. The charging powers for Batt. 1 and 2 and discharging power for Batt. 4 are both bounded.

In Fig. 15, the local bus voltages of the battery systems are higher than those of the ultracapacitor systems because the steady loads in the microgrid are provided by batteries and ultracapacitors only compensate the power fluctuations. The voltage deviation is caused by the battery currents supplying the steady component of loads connected at ultracapacitors.

\section{B. Case Study B. Resiliency to a Single Link Failure and Plug- and-Play Capability}

Based on the stability analysis in Section IV, even if there is a communication link loss, the rest of the communication network still forms a spanning tree and the results of (25) hold. Thus, the proposed control strategy is resilient to a single link failure and has plug-and-play capability.

Each battery ES system has a $70 \Omega$ constant load $(2.0629 \mathrm{~kW}$ at $380 \mathrm{~V}$ ), while each ultracapacitor ES system has a $140 \Omega$ constant load $(1.0315 \mathrm{~kW}$ at $380 \mathrm{~V})$. All other parameters are same as in the Case Study A.

1) Communication link failure: To verify resilience to a single link failure, the communication link between Battery 2 and Battery 3 is lost at $\mathrm{t}=60 \mathrm{~min}$ and hence the $\left\{S o C_{i}\right\}$ and $\left\{\bar{v}_{i}\right\}$ data cannot be exchanged. Figs. 17 and 18 show that despite the loss of communication, the SoCs are converging towards a common balanced state and the local bus voltages are maintained at the rated value.

2) Plug-and-play: Figs. 19 and 20 illustrate plug-and-play capability of the proposed control strategy. The Battery 2 converter disconnects at $\mathrm{t}=65 \mathrm{~min}$, both the converter output

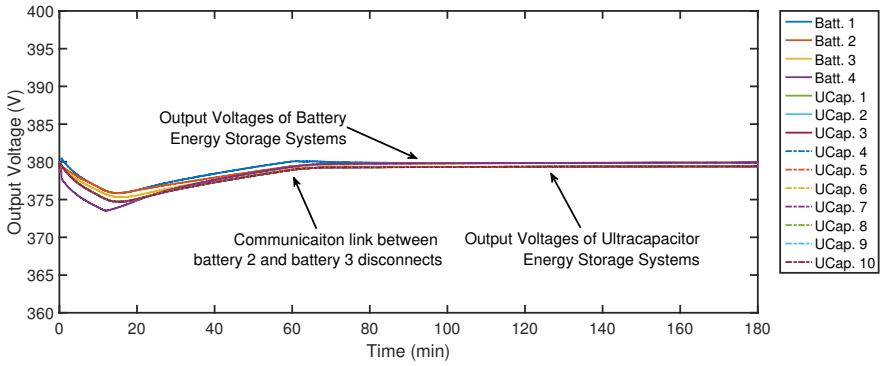

Fig. 18. Case Study B: 1) Local bus voltages.

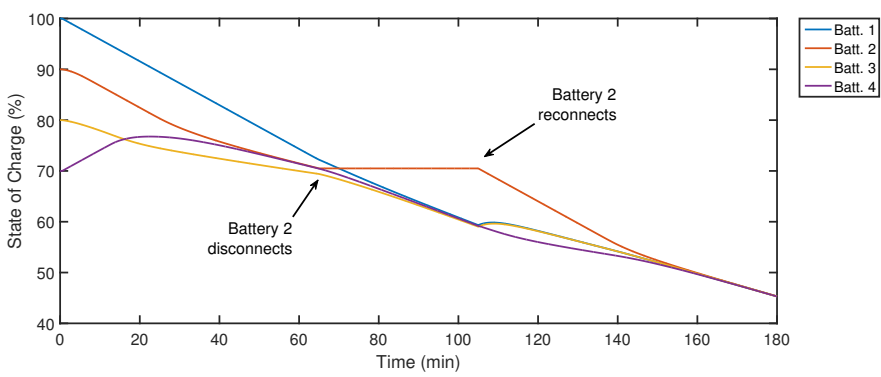

Fig. 19. Case Study B: 2) Battery SoC levels.

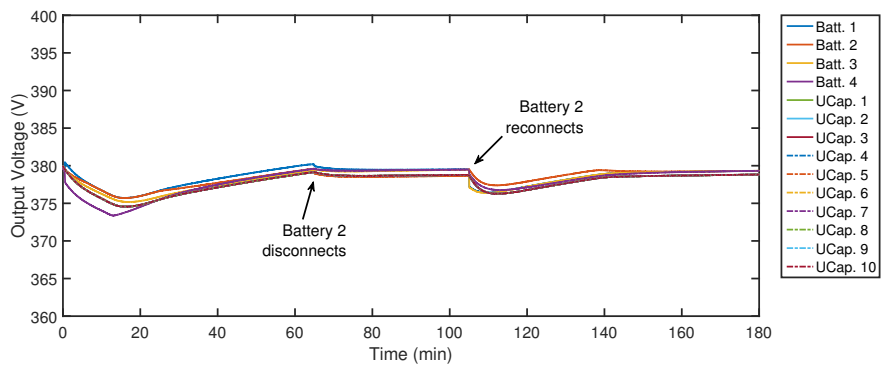

Fig. 20. Case Study B: 2) Local bus voltages.

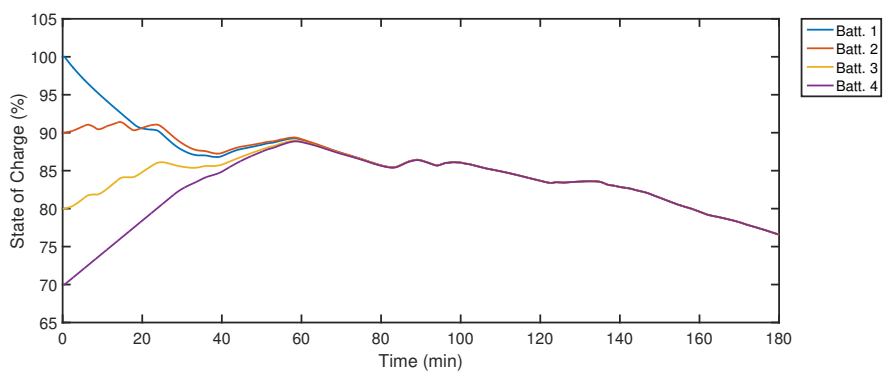

Fig. 21. Case Study C: SoCs.

and its communication are lost. Controllers automatically readjust the voltage regulation and balance power sharing between the batteries. Then, the converter and its communication link reconnect at $\mathrm{t}=105 \mathrm{~min}$. Correspondingly, the controllers restore the voltage regulation and energy balancing.

\section{The Microgrid with a PV Source}

To illustrate broader application of the proposed control strategy, a DC microgrid with a PV source is considered. The PV source interfaces with the microgrid through a DCDC converter and is regulated for the maximum power point 


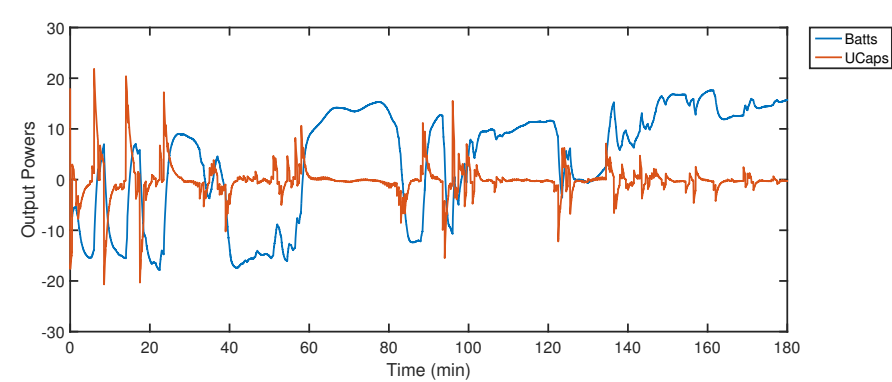

Fig. 22. Case Study C: Total output powers generated by batteries and ultracapacitors respectively.

tracking [34] for irradiance and temperature data with 1minute resolution, taken from the NREL Baseline Measurement Station in Colorado, from August 15, 2015 between 6:40 am and 9:40 am. Following the design procedure, the corner frequency is $60 \mathrm{~Hz}$ and the ultracapacitors are sized as $23.57 \mathrm{~F}$. The results are shown in Figs. 21 and 22.

\section{CONCLUSION}

In this paper, a cooperative and virtual capacitive control strategy was proposed for coordinating power sharing between heterogeneous ES devices distributed in a microgrid. The decentralized virtual capacitive control allocates different components of loads to the batteries and ultracapacitors respectively, based on the corner frequency. Additional decentralized control restores the ultracapacitor voltages. Moreover, the cooperative control, via a sparse communication network, regulates the local bus voltages and power sharing between batteries. The proposed control strategy is resilient to communication failure and provides plug-and-play capability. The control strategy offers advantages in terms of robustness, reliability and flexibility. Real-time digital simulation results verified performance of the proposed control approach.

\section{APPENDIX}

\section{STABILITY ANALYSIS}

The following lemmas are introduced to help to prove the stability.

Lemma 1 [5]: Assume that a graph $\mathcal{G}$ has a spanning tree and its Laplacian matrix $L$ is balanced. Then

$$
\lim _{s \rightarrow 0}\left(s I_{N}+L\right)^{-1}=\mathbf{Q}
$$

where $\mathbf{Q}$ is the averaging matrix, $\mathbf{Q} \in \mathbb{R}^{N \times N}$ whose elements are all equal to $1 / N$.

Lemma 2 [33]: Assume that vector $x \in \mathbb{R}^{N \times 1},\langle\mathbf{x}\rangle$ represents the average of all elements in the vector. Then,

$$
\mathbf{Q x}=\langle\mathbf{x}\rangle \mathbf{1}_{N},
$$

where $\mathbf{1}_{N} \in \mathbb{R}^{N \times 1}$ is the vector whose elements all equal one. Applying Lemma 1, the following limits are needed for obtaining the global steady state voltage.

$$
\begin{aligned}
& \lim _{s \rightarrow 0} \mathbf{G}_{\text {conv }}^{-1}=I_{N}, \lim _{s \rightarrow 0} \mathbf{r}_{d r p}=\mathbf{r}_{d r p}, \\
& \lim _{s \rightarrow 0} \mathbf{H}_{a v g}=\mathbf{Q}, \lim _{s \rightarrow 0} \mathbf{G}=\mathbf{G}, \lim _{s \rightarrow 0} s \mathbf{M}=\mathbf{M}^{0}, \\
& \lim _{s \rightarrow 0} Y_{\text {net }}=Y_{\text {net }}^{0}, \lim _{s \rightarrow 0} s \mathbf{H}=\mathbf{H}^{I},
\end{aligned}
$$

where $\mathbf{H}^{I}=\operatorname{diag}\left\{k_{\bar{v} I i}\right\}, \mathbf{M}^{0}=\operatorname{diag}\left\{-1 / C_{\text {batti }}^{\max }\right\}, I_{N}$ is an $N \times N$ identity matrix. Therefore, the steady state global voltage (24) can be written as

$$
\mathbf{v}^{s s}=\left(\mathbf{G} L \mathbf{M}^{0} Y_{n e t}^{0}+\mathbf{H}^{I} \mathbf{Q}\right)^{-1} \mathbf{H}^{I} \mathbf{v}_{m g} .
$$

Multiplying $\mathbf{G}^{-1}$ on both sides of (28) gives,

$$
\left(L \mathbf{M}^{0} Y_{n e t}^{0}+\mathbf{G}^{-1} \mathbf{H}^{I} \mathbf{Q}\right) \mathbf{v}^{s s}=\mathbf{G}^{-1} \mathbf{H}^{I} \mathbf{v}_{m g} .
$$

In the above procedure, assume that the proportional gains of SoC balancing control are negative, which implies that $\mathbf{G}^{-1}$ exists. Then, multiplying both sides of (29) by $\mathbf{Q}$ gives

$$
\left(\mathbf{Q} L \mathbf{M}^{0} Y_{n e t}^{0}+\mathbf{Q G}^{-1} \mathbf{H}^{I} \mathbf{Q}\right) \mathbf{v}^{s s}=\mathbf{Q G}^{-1} \mathbf{H}^{I} \mathbf{v}_{m g} .
$$

Since the Laplacian matrix is balanced, $\mathrm{Q} L=\mathbf{0}$ is always held. Thus, one can write (30) as

$$
\mathbf{Q G}^{-1} \mathbf{H}^{I} \mathbf{Q} \mathbf{v}^{s s}=\mathbf{Q G}^{-1} \mathbf{H}^{I} \mathbf{v}_{m g} \text {. }
$$

Implementing the Lemma 2 into the (31), the steady state voltage vector is

$$
\left\langle\mathbf{v}^{s s}\right\rangle\left\langle\mathbf{G}^{-1} \mathbf{H}^{I} \mathbf{1}_{N}\right\rangle \mathbf{1}_{N}=v_{m g}\left\langle\mathbf{G}^{-1} \mathbf{H}^{I} \mathbf{1}_{N}\right\rangle \mathbf{1}_{N} .
$$

Notice that the elements of both sides of (32) equal each other. Therefore, there are

$$
\left\langle\mathbf{v}^{s s}\right\rangle=v_{m g},
$$

which means that the average global voltage equals to the rated microgrid voltage. Rewriting (29) gives,

$$
\mathbf{G}^{-1} \mathbf{H}^{I} \mathbf{Q} \mathbf{v}^{s s}+L \mathbf{S o C} \mathbf{C}^{s s}=\mathbf{G}^{-1} \mathbf{H}^{I} \mathbf{v}_{m g} .
$$

Applying Lemma 2 into (34), one can obtain

$$
\mathbf{G}^{-1} \mathbf{H}^{I}\left\langle\mathbf{v}^{s s}\right\rangle \mathbf{1}_{N}+L \mathbf{S o C} \mathbf{C}^{s s}=v_{m g} \mathbf{G}^{-1} \mathbf{H}^{I} \mathbf{1}_{N} .
$$

Substituting (33) into (34), the following result is obtained,

$$
L \mathbf{S o C}^{s s}=\mathbf{0} .
$$

Since $L$ is designed to be balanced, $\mathbf{S o C}^{s s}$ is the right eigenvector of $L$ associated with $\lambda_{1}^{L}=0$. Furthermore, in accordance with the proof of Lemma 1 (see [5] ), $\mathbf{1}_{p}$ is the right eigenvector of the Laplacian matrix, $L$, associated with $\lambda_{1}^{L}=0$. Therefore,

$$
\mathbf{S o C}^{s s}=\varsigma \mathbf{1}_{p},
$$

where $\varsigma$ is a positive scalar. (37) indicates that the SoCs can converge to the same level.

Remark 6: From (28) and (29), the proportional control for the $\mathrm{SoC}$ balancing and the proportional integral control for the voltage restoration are sufficient for the control objectives (33) and (37). In [33], the proportional integral control for the SoC balancing and double integral control for the voltage restoration were applied. In addition, compared with [11], the method proposed in this paper needs to tune only the corner frequency, while the control system in [11], requires a communication link between battery and ultracapacitor to allocate the different components of load. Also, a set of proportional integral control parameters needs to be tuned to allocate the different components of load [11]. Thus, the method proposed in this paper simplifies the SoC balancing, the average voltage regulation and allocation of loads, when compared with the methods in [33] and [11]. 


\section{ACKNOWLEDGMENT}

R. Zhang would like to gratefully thank for a scholarship from the China Scholarship Council.

\section{REFERENCES}

[1] B. Zeng, J. Zhang, X. Yang, J. Wang, J. Dong, and Y. Zhang, "Integrated planning for transition to low-carbon distribution system with renewable energy generation and demand response," IEEE Transactions on Power Systems, vol. 29, no. 3, pp. 1153-1165, May 2014.

[2] A. Merabet, K. T. Ahmed, H. Ibrahim, R. Beguenane, and A. M. Y. M. Ghias, "Energy management and control system for laboratory scale microgrid based wind-pv-battery," IEEE Transactions on Sustainable Energy, vol. 8, no. 1, pp. 145-154, Jan 2017.

[3] R. Zhang, D. Chen, W. Yao, D. Ba, and X. Ma, "Non-linear fuzzy predictive control of hydroelectric system," IET Generation, Transmission Distribution, vol. 11, no. 8, pp. 1966-1975, 2017.

[4] G. Carpinelli, G. Celli, S. Mocci, F. Mottola, F. Pilo, and D. Proto, "Optimal integration of distributed energy storage devices in smart grids," IEEE Transactions on Smart Grid, vol. 4, no. 2, pp. 985-995, June 2013.

[5] B. Ali, N. Vahidreza, D. Ali, and L. Frank, Cooperative Synchronization in Distributed Microgrid Control. Cham, Switzerland: Springer, 2017.

[6] M. Kumar, S. C. Srivastava, and S. N. Singh, "Control strategies of a dc microgrid for grid connected and islanded operations," IEEE Transactions on Smart Grid, vol. 6, no. 4, pp. 1588-1601, July 2015.

[7] P. Prabhakaran, Y. Goyal, and V. Agarwal, "Novel nonlinear droop control techniques to overcome the load sharing and voltage regulation issues in dc microgrid," IEEE Transactions on Power Electronics, vol. PP, no. 99, pp. 1-1, 2017.

[8] S. Augustine, M. K. Mishra, and N. Lakshminarasamma, "Adaptive droop control strategy for load sharing and circulating current minimization in low-voltage standalone dc microgrid," IEEE Transactions on Sustainable Energy, vol. 6, no. 1, pp. 132-141, Jan 2015.

[9] V. Nasirian, A. P. Yadav, F. L. Lewis, and A. Davoudi, "Distributed assistive control of power buffers in dc microgrids," IEEE Transactions on Energy Conversion, vol. 32, no. 4, pp. 1396-1406, Dec 2017.

[10] K. Strunz, E. Abbasi, and D. N. Huu, "Dc microgrid for wind and solar power integration," IEEE Journal of Emerging and Selected Topics in Power Electronics, vol. 2, no. 1, pp. 115-126, March 2014.

[11] T. Morstyn, B. Hredzak, and V. G. Agelidis, "Cooperative multi-agent control of heterogeneous storage devices distributed in a dc microgrid," IEEE Transactions on Power Systems, vol. 31, no. 4, pp. 2974-2986, July 2016.

[12] X. Qiu, T. A. Nguyen, and M. L. Crow, "Heterogeneous energy storage optimization for microgrids," IEEE Transactions on Smart Grid, vol. 7, no. 3, pp. 1453-1461, May 2016.

[13] P. Kreczanik, P. Venet, A. Hijazi, and G. Clerc, "Study of supercapacitor aging and lifetime estimation according to voltage, temperature, and rms current," IEEE Transactions on Industrial Electronics, vol. 61, no. 9, pp. 4895-4902, Sept 2014.

[14] D. B. W. Abeywardana, B. Hredzak, V. G. Agelidis, and G. D. Demetriades, "Supercapacitor sizing method for energy-controlled filterbased hybrid energy storage systems," IEEE Transactions on Power Electronics, vol. 32, no. 2, pp. 1626-1637, Feb 2017.

[15] N. Korada and M. K. Mishra, "Grid adaptive power management strategy for an integrated microgrid with hybrid energy storage," IEEE Transactions on Industrial Electronics, vol. 64, no. 4, pp. 2884-2892, April 2017.

[16] Y. Gu, W. Li, and X. He, "Frequency-coordinating virtual impedance for autonomous power management of dc microgrid," IEEE Transactions on Power Electronics, vol. 30, no. 4, pp. 2328-2337, April 2015.

[17] Q. Xu, X. Hu, P. Wang, J. Xiao, P. Tu, C. Wen, and M. Y. Lee, "A decentralized dynamic power sharing strategy for hybrid energy storage system in autonomous dc microgrid," IEEE Transactions on Industrial Electronics, vol. 64, no. 7, pp. 5930-5941, July 2017.

[18] Y. Wang, X. Lin, Y. Kim, Q. Xie, M. Pedram, and N. Chang, "Singlesource, single-destination charge migration in hybrid electrical energy storage systems," IEEE Transactions on Very Large Scale Integration (VLSI) Systems, vol. 22, no. 12, pp. 2752-2765, Dec 2014.

[19] Q. Xie, Y. Wang, Y. Kim, M. Pedram, and N. Chang, "Charge allocation in hybrid electrical energy storage systems," IEEE Transactions on Computer-Aided Design of Integrated Circuits and Systems, vol. 32, no. 7, pp. 1003-1016, July 2013.
[20] X. Lu, K. Sun, J. M. Guerrero, J. C. Vasquez, and L. Huang, "Doublequadrant state-of-charge-based droop control method for distributed energy storage systems in autonomous dc microgrids," IEEE Transactions on Smart Grid, vol. 6, no. 1, pp. 147-157, Jan 2015.

[21] T. Dragičević, J. M. Guerrero, J. C. Vasquez, and D. Skrlec, "Supervisory control of an adaptive-droop regulated dc microgrid with battery management capability," IEEE Transactions on Power Electronics, vol. 29 , no. 2, pp. 695-706, Feb 2014.

[22] H. Zhang, S. Kim, Q. Sun, and J. Zhou, "Distributed adaptive virtual impedance control for accurate reactive power sharing based on consensus control in microgrids," IEEE Transactions on Smart Grid, vol. 8, no. 4, pp. 1749-1761, July 2017.

[23] J. Xiao, P. Wang, L. Setyawan, and Q. Xu, "Multi-level energy management system for real-time scheduling of dc microgrids with multiple slack terminals," IEEE Transactions on Energy Conversion, vol. 31, no. 1, pp. 392-400, March 2016.

[24] D. I. Brandao, T. Caldognetto, F. P. Marafão, M. G. Simões, J. A. Pomilio, and P. Tenti, "Centralized control of distributed single-phase inverters arbitrarily connected to three-phase four-wire microgrids," IEEE Transactions on Smart Grid, vol. 8, no. 1, pp. 437-446, Jan 2017

[25] A. Khorsandi, M. Ashourloo, and H. Mokhtari, "A decentralized control method for a low-voltage dc microgrid," IEEE Transactions on Energy Conversion, vol. 29, no. 4, pp. 793-801, Dec 2014.

[26] N. M. Dehkordi, N. Sadati, and M. Hamzeh, "Fully distributed cooperative secondary frequency and voltage control of islanded microgrids," IEEE Transactions on Energy Conversion, vol. 32, no. 2, pp. 675-685, June 2017.

[27] T. Morstyn, B. Hredzak, and V. G. Agelidis, "Control strategies for microgrids with distributed energy storage systems: An overview," IEEE Transactions on Smart Grid, vol. PP, no. 99, pp. 1-1, 2016.

[28] Y. Zhang and Y. W. Li, "Energy management strategy for supercapacitor in droop-controlled dc microgrid using virtual impedance," IEEE Transactions on Power Electronics, vol. 32, no. 4, pp. 2704-2716, April 2017.

[29] L. Meng, T. Dragicevic, J. Roldán-Pérez, J. C. Vasquez, and J. M. Guerrero, "Modeling and sensitivity study of consensus algorithm-based distributed hierarchical control for dc microgrids," IEEE Transactions on Smart Grid, vol. 7, no. 3, pp. 1504-1515, May 2016.

[30] T. Kim and W. Qiao, "A hybrid battery model capable of capturing dynamic circuit characteristics and nonlinear capacity effects," IEEE Transactions on Energy Conversion, vol. 26, no. 4, pp. 1172-1180, Dec 2011.

[31] P. Sanchis, A. Ursaea, E. Gubia, and L. Marroyo, "Boost dc-ac inverter: a new control strategy," IEEE Transactions on Power Electronics, vol. 20, no. 2, pp. 343-353, March 2005

[32] F. L. Lewis, H. Zhang, K. Hengster-Movric, and A. Das, Cooperative Control of Multi-Agent Systems. London: Springer-Verlag, 2014.

[33] T. Morstyn, B. Hredzak, G. D. Demetriades, and V. G. Agelidis, "Unified distributed control for dc microgrid operating modes," IEEE Transactions on Power Systems, vol. 31, no. 1, pp. 802-812, Jan 2016.

[34] T. Morstyn, B. Hredzak, R. P. Aguilera, and V. G. Agelidis, "Model predictive control for distributed microgrid battery energy storage systems," IEEE Transactions on Control Systems Technology, vol. PP, no. 99, pp. $1-8,2017$. 